\title{
Interstellar media in other galaxies
}

\section{from a correspondent}

A recent international workshop at the European Space Agency's (ESA) Villafranca tracking station (Vilspa) near Madrid brought together those studying the interstellar medium in our own galaxy and those concerned with extragalactic problems, in particular the quasar absorbtion lines. Data from both the International Ultraviolet Explorer (IUE) satellite, which is controlled from Vilspa during its European observing shift, and Copernicus, as well as from ground based telescopes were discussed. The IUE has just begun making important observation of interstellar media and, with the demise of Copernicus at the end of 1980 , will be the prime instrument for interstellar studies until the launch of Space Telescope.

Ted Snow (Colorado) reviewed work on the abundances of elements and their depletion in the interstellar gas as a result of intensive studies of particular sightlines with the Copernicus ultraviolet spectrometer. While gas-phase abundances of some species, in particular carbon and oxygen, are little depleted and this sets limits on grain composition, the refractory elements are more highly depleted, consistent with either grain condensation in a high-density environment or preferential accretion of these species onto grain mantles by dynamic processes in interstellar clouds. Stuart Pottasch (Groningen) noted that, for a few species, the existence or degree of depletion depended on the cosmic abundances adopted and these were themselves not beyond controversy. Nolan Walborn (Cerro Tololo) reported some recent work on the dynamics of the interstellar gas in the $\eta$ Carina nebula; relative velocities up to $550 \mathrm{~km} \mathrm{~s}^{-1}$ are seen. Ed Jenkins (Princeton) discussed the presence of highly ionized gas in the plane of the galaxy. It appears that Ovı lines define a component with scale height above the galactic plane of $\sim 300$ pc and a velocity dispersion of $25 \mathrm{~km} \mathrm{~s}^{-1}$. Their strength is correlated with reddening by dust. By contrast there is still some controversy whether the CIV and Sirv lines are truly interstellar. There seemed some evidence that the latter, at least, was in part circumstellar. Gordon Bromage (Chilton) claimed that Nv lines were not seen in IUE data covering sight-lines to halo stars which rise out of the galactic plane so that the Nv distribution looks similar to that of OVI with a scale height much smaller than that of the lower temperature Civ near $3 \mathrm{kpc}$. Max Pettini (Herstmonceux) noted, however, that he had one case where $\mathrm{Nv}$ was seen in a halo star and he added that the three-time ionized carbon abundance may be lower in the plane than just outside it.

Klaas de Boer (Washburn) reviewed the recent exciting work by $I U E$ on the galactic halo with emphasis on sight-lines to the Magellanic Clouds. There is some uncertainty if gas far from the plane corotates with the plane but, if so, the density falls by a factor of 10 over $10 \mathrm{kpc}$ and one cloud covering 7 square degrees in the direction of the Large Cloud may contain 300,000 solar masses. There is component structure in $\mathrm{OI}$ and Fell but the Silv and, possibly, Civ profiles suggested the halo has a smoother, less cloudy structure than the plane. There was, however, some discussion as to whether the direction to the Magellanic Clouds (where most of the IUE data exists) is typical of our galaxy. Claudine Laurent (Verrieres-le-Buisson) commented also that the existence of a similar halo around the Small Cloud was in doubt as she had data showing no CIV or Silv in an SMC star which, unlike other cases observed to date, did not have its own Hıl region. Chris Blades (Chilton) reported ground-based optical data from the Anglo-Australian Telescope (AAT) and found that the ratio of the neutral sodium column to dust reddening in the Small Cloud was 5 times smaller than that in our own galaxy.

Michael Penston (Vilspa) also showed AAT data of optical interstellar lines in other galaxies using galactic nuclei or supernovae as background probes. He confirmed earlier reports of Call absorption in the Magellanic Stream. In discussion, the point was made that the (by necessity) moderate resolution on optical interstellar lines in other galaxies is similar in quality to the type of data that was available for our own galaxy in the 1940s and 1950s. While it is valid to analyse the galactic and extragalactic doublet ratios is similar ways and show that turbulent motions in interstellar media are generally characterised by velocity dispersions similar to that in our own galaxy, it was agreed that it is dangerous to deduce column densities from such material because large columns can be 'hidden' in components with low velocity dispersion. Alec Boksenberg (London) reviewed the current status of work on quasar absorptions lines. The absorption systems with metal lines can arise in galactic halos if these have radii of 100 $\mathrm{kpc}$. The 'Lyman- $\alpha$-only' absorption systems are not clustered like galaxies and suggest the presence of intergalactic clouds of primordial material.

On the theoretical side, Jo Silk (Berkeley) discussed other less direct evidence for intergalactic clouds including deduction from theories of galaxy formation. He proposed that slow self-regulating star-formation takes place in these clouds prior to their merging into galaxies as we know them. Joel Bregman (New York) described his 'galactic fountain' model of galactic haloes, in which hot gas of temperature $T_{\mathrm{o}}$ at the base of the galactic corona rises, cools and turns into clouds which fall back towards the plane. Coronae of the right size are produced if $T_{0}$ is in the range one to six million degrees. A total mass of $10^{10}$ solar masses is required in these galactic haloes. understood part of the electron transport system. Recent reports from Winget (Cincinnati) on reconstitution of this system using an isolated manganeseprotein were not substantiated by other workers. For example, the reconstituted system described by Nakatani et al. (Imperial College London) requires a catalase-like protein rather than a manganese protein. Also, the NMR measurements of Wyrdzinski and Govindjee (Urbana) that appeared to reflect changes in the redox state of manganese were challenged by Sharp and Yocum (Ann Arbor, Michigan). Nevertheless, materials for the solution of the 'water-splitting problem' are accumulating. Oxygen-evolving preparations with increased specific activity were described by Stewart (Cambridge,
England), inverted vesicles, that is, with the water oxidation system on the outside, were described by Albertsson (Lund), and Bishop (Corvallis, Oregon) has discovered mutants with defective water-oxidation systems. We cannot yet be 100 per cent sure, however, according to Metzner (Tubingen), that it is $\mathrm{H}_{2} \mathrm{O}$ and not $\mathrm{CO}_{2}$ that is releasing oxygen!

The sessions on chloroplast metabolism 